\title{
Animal Reservoirs and Hosts for Emerging Alpha- and Betacoronaviruses
}

\author{
Ria R. Ghai, Ann Carpenter, Meghan K. Herring, Amanda Y. Liew, Krystalyn B. Martin, Susan I. \\ Gerber, Aron J. Hall, Jonathan M. Sleeman, Sophie VonDobschuetz and Casey Barton Behravesh \\ 1 U.S. Centers for Disease Control and Prevention, Atlanta, GA, United States (R. Ghai, A. Carpenter, M. \\ Herring, A. Liew, K. Martin, S. Gerber, A Hall, C Barton Behravesh) \\ 2 Emory University, Atlanta, GA, United States (M. Herring, A. Liew, K. Martin) \\ 3 U.S. Geological Survey National Wildlife Health Center, Madison, WI, United States (J. Sleeman) \\ 4 Food and Agriculture Organization of the United Nations, Rome, Italy (S. VonDobschuetz)
}

Running Title: Animal hosts for emerging alpha and betacoronaviruses

Article Summary: A review of coronaviruses in wildlife, livestock, and companion animals, and comprehensive data on receptor usage, hosts, and clinical presentation of 15 previously or currently emerging alpha-or beta-coronaviruses in people and animals.

\begin{abstract}
The ongoing global pandemic caused by coronavirus disease 2019 (COVID-19) has once again demonstrated the significance of the Coronaviridae family in causing human disease outbreaks. As SARS-CoV-2 was first detected in December 2019, information on its tropism, host range, and clinical presentation in animals is limited. Given the limited information, data from other coronaviruses may be useful to inform scientific inquiry, risk assessment and decision-making. We review the endemic and emerging alpha- and betacoronavirus infections of wildlife, livestock, and companion animals, and provide information on the receptor usage, known hosts, and clinical signs associated with each host for 15 coronaviruses discovered in people and animals. This information can be used to guide implementation of a One Health approach that involves human health, animal health, environmental, and other relevant partners in developing strategies for preparedness, response, and control to current and future coronavirus disease threats.
\end{abstract}

Keywords: Emerging infectious diseases; coronaviruses; COVID-19; SARS-CoV; SARS-CoV-2; MERS-CoV; zoonotic diseases

\section{Background}

Coronaviruses (CoVs) are a family of RNA viruses whose large genomes, propensity for mutation, and frequent recombination events have resulted in a diversity of strains and species that are capable of rapid adaptation to new hosts and ecological environments (1). This viral plasticity has garnered widespread concern over their zoonotic potential and the consequences of new emergence events in both human and animal populations. The emergence of a new strain of severe acute respiratory syndrome (SARS) coronavirus, SARS-CoV-2, which causes coronavirus disease 2019 (COVID-19) has once again demonstrated the significance of the Coronaviridae family in causing human disease outbreaks. SARS-CoV-2, a novel betacoronavirus, was first identified in human patients from Wuhan, China in December 2019 and has resulted in a global pandemic, an unprecedented public health emergency, and untold economic and societal repercussions worldwide. Like the 2002-03 SARS epidemic, the first cluster of COVID-19 cases were suspected to be associated with a live animal market where hundreds of animal species were sold, including wildlife, livestock, companion animals, and seafood (2). Zoonotic transmission of SARS-CoV-2 from 
horseshoe bats (genus Rhinolophus) to humans is likely, perhaps through a yet unidentified intermediate host species (3).

While COVID-19 is novel in the breadth of the human outbreak, a number of pathogenic alphaand betacoronaviruses have shown similar patterns of emergence previously. As early as the 1930s, coronaviruses pathogenic to livestock, companion animals, and laboratory animals were identified (4). During the 1960s, two human coronaviruses, HCoV-229E and -OC43, were discovered in patients with common colds $(5,6)$. While there is speculation that HCoV-OC43 may also have emerged through a global pandemic in the late 1800s (7), the 2002-03 SARS outbreak is the first accepted global epidemic caused by a coronavirus. The SARS epidemic triggered research within this viral family (4). This research led to the discovery of two new human coronaviruses, HCoV-NL63 and -HKU1 $(8,9)$. HCoV-229E, -OC43, -NL63 and -HKU1 are now accepted as globally endemic "common cold" species that are typically associated with mild-to-moderate respiratory illness. In 2012, the most deadly human coronavirus to date was discovered in the Arabian peninsula: Middle East respiratory syndrome coronavirus (MERS-CoV) (10). A cumulative body of research on these and other coronaviruses has shown that most alpha and betacoronaviruses infecting humans have come from animal hosts, and that both historic patterns and coronavirus biology establish an urgent ongoing threat to human and animal health (11). While coronaviruses are divided into four viral genera, namely alpha-, beta-, gamma-, and deltacoronaviruses, here we focus on alpha- and betacoronaviruses since all known human coronaviruses are from these genera, and they may therefore pose an increased risk of causing future human pandemics.

This review is intended to compile data to inform a One Health approach to combatting emerging alpha- and betacoronaviruses. One Health is a collaborative, multisectoral, and transdisciplinary approach - working at the local, regional, national, and global levels - with the goal of achieving optimal health outcomes recognizing the interconnection between people, animals, plants, and their shared environment (12). For example, in Qatar, a One Health approach for MERS$\mathrm{CoV}$ prevention and control has been implemented since early in the outbreak, and is associated with improvements in coordination, joint outbreak response rates, and diagnostic capacity (13). Similarly, in the United States, establishment of the One Health Federal Interagency COVID-19 Coordination Group has been instrumental in ensuring an efficient and coordinated all-of-government response by creating a mechanism to communicate, share timely updates, and align messaging (14). More generally, the One Health approach is endorsed as an effective means of combatting zoonotic diseases internationally by the Tripartite international health organizations, consisting of the Food and Agriculture Organization of the United Nations (FAO), the World Health Organization (WHO), and the World Organisation for Animal Health (OIE) (15).

As with other zoonotic diseases, effective implementation of a One Health approach for emerging coronaviruses requires an understanding of the transmission dynamics and human and animal hosts associated with the pathogen. This review therefore summarizes information from other coronavirus emergence events, which may be useful in identifying trends, establishing baselines, and informing decision-making using a One Health approach around the current COVID-19 pandemic and future emerging coronavirus threats. Specifically, we provide information on the receptor used by each current or previously emerging coronavirus since tropism can help predict host susceptibility (Table 1), all known hosts of each coronavirus and their host category (i.e., reservoir, intermediate, spillover, susceptible through experimental infection, or non-susceptible through experimental infection; Table 2), and clinical signs associated with coronavirus infection (Table 3).

\section{Emerging Coronaviruses and Wildlife}

More than $70 \%$ of zoonotic emerging infectious diseases in humans are caused by pathogens with a wildlife origin (16). A number of mammalian orders are now known to host coronaviruses, including carnivores, lagomorphs, non-human primates, ungulates and rodents (4). However, the attention has focused on Chiroptera (bats), which are hypothesized to be the origin host for all alphaand betacoronaviruses, and therefore all human coronaviruses (Table 2) $(1,4)$. 
After rodents, bats are the second most diverse and abundant mammalian order, comprising $20 \%$ of all mammalian biodiversity worldwide (17). In the last two decades, research has intensified to determine why bats harbor more zoonotic diseases than other mammalian taxa, including pathogens that result in high-consequence human emerging infectious diseases like Ebola and Marburg filoviruses, Nipah and Hendra paramyxoviruses, and SARS-CoV, SARS-CoV-2, and MERS$\mathrm{CoV}$ (18). Their gregariousness, sympatry with mixed species assemblages in roosts, long lifespan relative to size, absence of clinical illness due to viral infection, and increased metabolic rate likely all contribute to why bats are reservoirs to many viral pathogens (18).

Coronavirus richness and diversity detected from bats far exceeds those of other mammalian orders, with at least 11 of 18 chiropteran families across six continents testing positive for one or more coronavirus species (4). Recently, a study surveying the diversity of wildlife coronaviruses across global disease "hotspots" identified 100 distinct viruses, of which 91 were detected in bats (11). Interestingly, the study discovered that patterns of coronavirus diversity mirrored bat diversity and evolutionary history, reinforcing the idea that bats are the predominant reservoir of zoonotic and emerging coronaviruses (11). Based on extrapolations made in the same study, the authors predict that bats harbor $\sim 3,204$ coronaviruses, the majority of which remain undiscovered (11).

Despite the risks associated with bat-origin coronaviruses, bats play integral roles in ecosystems, including insect suppression through predation, prey for numerous predators, pollinators for economically and ecologically important plants, and seed dispersal for countless tropical trees and shrubs (19). Therefore, mitigating the risks of future emergence events from bats should focus on minimizing close interaction between people and bats and other wildlife, such as reductions to or cessation of wildlife sales at wet markets, wildlife hunting, and encroachment on wildlife habitat.

While further bat research may help us understand the origins of coronaviruses, other wildlife species are intermediate hosts for human emerging coronaviruses. Intermediate hosts may not only add complexity to coronavirus transmission dynamics, but may also amplify viral spillover to new hosts by closings gaps in interaction frequency between species, and by increasing transmissibility and/or infectiousness through viral adaptation (20). A canonical example is SARS-CoV, whose intermediate host is accepted to be palm civets (21). In this instance, close interaction between people and civets sold through wildlife markets likely facilitated transmission to humans, while passage and ongoing recombination in civet intermediate hosts is believed to have played a critical role in facilitating human receptor tropism $(20,22)$ (Table 1$)$.

Some wildlife species are at risk of human coronavirus spillover. Wild great apes, all species of which are endangered, are one taxonomic group likely vulnerable to spillover from humans, at least in part because they are our closest living relatives. A number of documented respiratory outbreaks that resulted in clinical signs ranging from mild illness to death in chimpanzee and gorilla populations originate from a human source $(23,24)$. Perhaps most significantly, the human betacoronavirus $\mathrm{HCoV} \mathrm{OC43}$ was discovered as the causative agent of mild-to-moderate respiratory illness among wild chimpanzees in Côte D'Ivoire in late 2016 and early 2017 (Table 2) (25), suggesting their susceptibility to human coronaviruses. As the COVID-19 pandemic continues, there is concern that susceptible wildlife such as great apes may be exposed to the virus through human contact, resulting in establishment of a new host reservoir which could pose a risk of perpetuating enzootic transmission and zoonotic transmission into recovering human populations.

Wildlife infections of SARS-CoV-2 have already occurred - the first natural infection of SARSCoV-2 in a wild animal, and the first confirmed animal cases in the United States, were in captive tigers $(n=5)$ and lions $(n=3)$ at a zoo in New York City (26). Unlike most other asymptomatic animal cases reported previously, the large cats demonstrated respiratory clinical signs that included coughing and wheezing, but ultimately made a full recovery (26) (Table 3). SARS-CoV-2 infection in captive wild felids highlights the complex interactions people may have with wildlife, including the potential for human-to-wildlife transmission. Given these interlinkages, framing risk using a One Health approach may more comprehensively address both the socio-economic and environmental drivers of disease emergence leading to potentially novel, mutually beneficial solutions. For example, risks could be reduced by regulation of wildlife trade and markets and development of sanitary 
standards, which would not only protect public health and animal health, but also result in positive wildlife conservation outcomes.

\section{Emerging Coronaviruses and Livestock}

Some coronaviruses naturally infect livestock and can have devastating economic consequences, such as swine acute diarrhea syndrome (SADS) coronavirus, porcine epidemic diarrhea virus (PEDV), and Betacoronavirus 1. While recent studies suggest that pigs are not susceptible hosts for SARS-CoV-2 infection $(27,28)$, pigs are a common host for alpha- and betacoronaviruses, with six different viral species causing disease (29) (Table 2). Of these, the enteric alphacoronaviruses PEDV and SADS-CoV (a strain of the Rhinolophus bat coronavirus HKU2), are considered re-emerging and emerging, respectively (29). While PEDV was first detected in China in the 1970s, a highly pathogenic variant caused considerable losses to the United States pork industry in 2013-14 (30). SADS-CoV is highly pathogenic in swine and was first detected in Guangdong province in 2016-17, causing the death of nearly 25,000 piglets (31) (Table 3). SADS-CoV emerged within $100 \mathrm{~km}$ of the accepted locale of the SARS index case, and like SARS-CoV and SARS-CoV-2, SADS-CoV is suspected to originate in horseshoe bats (Rhinolophus spp.) (31) (Table 2). Unlike SARS-CoV and SARS-CoV-2, however, SADS$\mathrm{CoV}$ has not been detected outside of China (29).

Among betacoronaviruses, a strain of Betacoronavirus 1also infects pigs (29). Porcine hemagglutinating encephalomyelitis virus (PHEV) has been circulating for decades and causes rapid death in piglets (29) (Table 3). Unlike other coronaviruses, Betacoronavirus 1 is a unique species complex, in that its distinct strains are host-specific to a range of different species, including wild and domestic ungulate, rabbits, and canines $(20,32)$. Perhaps the most well-studied strain of Betacoronavirus 1is bovine coronavirus $(\mathrm{BCoV})$, which is of economic significance as it can be associated with a suite of clinical disease in calves and cattle, including calf diarrhea, winter dysentery, and respiratory infection (32) (Table 3). BCoV also infects a number of other livestock species including horses, sheep, and camels $(20,32)$ (Table 2$)$.

Livestock have also been intermediate hosts in the emergence of three human coronaviruses. First, an unknown ungulate species, speculated to be cattle, is accepted as the intermediate host of human coronavirus OC43 (HCoV-OC43) $(7,33)$, a strain of Betacoronavirus 1 (Table 2). Based on molecular clock calculations, $\mathrm{HCoV}-\mathrm{OC} 43$ is predicted to have jumped from livestock to humans around 1890, a timeframe coincident with pandemics of respiratory disease in both cattle (which resulted in widespread culling) and people (although this outbreak is historically attributed to influenza) (7). Dromedary camels are accepted as established hosts of MERS-CoV and are thought to be associated with the emergence of $\mathrm{HCoV}-229 \mathrm{E}$ in people based on closely related viruses found in camelids (34) (Table 2). Dromedary camels inhabit the Middle East and Northern Africa and comprise $90 \%$ of extant camels on earth. In much of their range, dromedaries are an important livestock species that are used as racing animals, working animals, and for their milk, meat, and hides.

Livestock can also be spillover hosts of human coronavirus infection. Following the 2002-03 SARS outbreak, a study conducted on farms in Xiqing County, China, tested livestock (pigs, cattle, chickens, and ducks) and companion animals (dogs and cats), leading to discovery of one pig that was positive for SARS-CoV by antibody test and RT-PCR (35) (Table 2). A larger and more complex series of livestock outbreaks of SARS-CoV-2 has been ongoing since April 2020, as 40 mink farms in the Netherlands, four in Denmark, one in Spain, and two in the United States have identified mink positive for SARS-CoV-2. In all outbreaks, mink were suspected to be initially infected by COVID19-positive farm workers (36). In the Netherlands, unpublished reports have identified plausible instances of spillback from mink to humans in ongoing investigations (26). National surveillance and control efforts have been implemented in each country, which have identified other SARS-CoV-2 positive species living on mink farms, including 7 barn cats in the Netherlands (out of 24 sampled), and $1 \mathrm{dog}$ in Denmark. Both countries have implemented mandatory reporting of any positive animals, and depopulation of affected farms. In the Netherlands to-date, more than 1.5 million mink have been culled and a moratorium has been placed on the mink industry, which highlights the 
necessity of early and coordinated One Health action to prevent bi-directional transmission of zoonotic diseases (26).

\section{Emerging Coronaviruses and Companion Animals}

Companion animals are an important part of many households and can improve both the physical and mental well-being of their owners (37). In the United States, approximately 71.5 million households (57\%) own at least one companion animal (38). Among households with companion animals, dogs (67\%) and cats (44\%) are the most commonly owned (38). Despite the many benefits of pet ownership, close interactions with pets pose risks for zoonotic disease transmission. Zoonotic diseases that are spread between people and companion animals include rabies, salmonellosis, campylobacteriosis, and hookworms, among others $(37,39,40)$. Companion animals are estimated to be a source of more than 70 human diseases (41), and the burden of zoonotic diseases attributed to interactions with companion animal is substantial. For example, rabies kills an estimated 59,000 people per year globally, and $99 \%$ of human rabies cases originate from rabid dogs (40).

A number of common coronaviruses have been discovered in companion animals, although none of the coronaviruses that are endemic in companion animal populations are zoonotic. One of the most common respiratory diseases in dogs is canine infectious respiratory disease (CIRD), or kennel cough, which typically causes cough and nasal discharge in puppies and dogs $(42,43)$. While kennel cough can be caused by a number of pathogens, most frequently the bacterium Bordetella bronchiseptica, canine respiratory coronavirus $(\mathrm{CRCoV})$ is a contributing pathogen to this syndrome $(42,44)$ (Table 1). CRCoV is believed to originate from $B C o V$, either through a common ancestor, host variant, or a host species shift, and is therefore considered a strain of Betacoronavirus-1 $(42,44)$. Regardless of how $\mathrm{CRCoV}$ and $\mathrm{BCoV}$ are genetically related, experimental studies shown that dogs challenged with $\mathrm{BCoV}$ can become infected and transmit the virus to other dogs, though they do not exhibit clinical signs of disease (45) (Table 2 and 3).

Canine enteric coronavirus $(\mathrm{CCoV})$ is an alphacoronavirus often associated with mild enteritis in puppies and dogs, especially in group housing situations (46). In 2005, however, a novel, highly pathogenic variant strain of CCoV-II, CB/05, was first identified (47) (Table 2). This new variant is now pantropic, and has a mortality rate of up to $100 \%$ in isolated outbreaks in puppies (47) (Table 3). Due to its increased pathogenicity and changes in tissue tropism, $\mathrm{CCoV}$ is considered an emerging pathogen (46).

While $\mathrm{CCoV}$ is generally considered to be specific to dogs, cats experimentally challenged with the virus can be infected with $\mathrm{CCoV}$ and mount an anamnestic response to further exposure, although they do not develop clinical signs of illness (48) (Table 3). Indeed, while there exist two serotypes of feline coronavirus (FCoV), FCoV type I and FCoV type II, type II is hypothesized to have originated from a recombination event between $\mathrm{FCoV}$ type $\mathrm{I}$ and $\mathrm{CCoV}$, which suggests co-infections of coronaviruses among companion animals may yield opportunity for the emergence of new disease (49).

Companion animals may also act as spillover hosts for human coronaviruses. A study following the 2002-03 SARS outbreak discovered that pet cats living in a Hong Kong apartment complex were naturally infected with SARS during the epidemic (50). Following the epidemic, challenge experiments in cats and ferrets found that both species could be experimentally infected and transmit the infection to naive animals of the same species they were housed with (50) (Table 2). In this experiment, cats did not demonstrate clinical signs of illness, although ferrets became lethargic, developed conjunctivitis, and died on days 16 and 21 post-infection. However, unlike human cases, there was no evidence that SARS-CoV-associated pneumonia was a cause of death (Table 3). Rather, the main findings in deceased ferrets were marked hepatic lipidosis and emaciation (50).

Companion animals, specifically dogs and cats, are among the most commonly infected groups of animals in the ongoing COVID-19 pandemic. Natural cases of suspected human-to-animal transmission have been confirmed in dogs from Hong Kong and the United States (51), and in cats in China, Hong Kong, the United States, and several European countries (26). Experimental challenge studies additionally suggest that like SARS-CoV, a number of companion animals, including cats, 
ferrets, and golden hamsters and are all susceptible to SARS-CoV-2 infection under laboratory conditions $(27,28,52-54)$ (Table 2). Furthermore, studies in cats, hamsters and ferrets show they are capable of both direct and indirect transmission to healthy conspecifics in experimental settings (27, $28,53,54)$, which underscores the need for infection prevention and control practices between people to companion animals.

The global prevalence of companion animal ownership underscores the need for better understanding of pathogens, such as coronaviruses, that can infect pets. As companion animals harbor endemic coronaviruses and may also be at risk of spillover for some human zoonotic coronaviruses, there is potential for coronavirus recombination events and new viral emergence to occur within these hosts. Therefore, ensuring that people understand how to safely interact with their companion animals is essential for ensuring that both people and companion animals stay healthy while also protecting animal welfare.

\section{Conclusions}

A considerable number of mammalian species, including wildlife, livestock, and companion animals, are susceptible to infection with alpha- and betacoronaviruses. The propensity of alpha- and betacoronaviruses to jump to new hosts, coupled with their relatively large host ranges, suggests that a One Health approach should be used to develop strategies to mitigate the effects of current and future coronavirus emergence events. During the COVID-19 pandemic, One Health collaboration between public health and veterinary sectors has already bolstered critical healthcare resources and infrastructure, leading to improvements in diagnostic testing capacity and human resource availability (55). In the United States, the One Health Federal Interagency COVID-19 Coordination Group has developed risk communication and messaging for companion animals, livestock, and wildlife and has been instrumental in coordinating joint outbreak response and diagnostic testing in animals. As these examples highlight, integration of the One Health approach into preparedness planning, joint epidemiological investigations, surveillance, laboratory diagnostics, risk assessment and field research is not only warranted, but necessary to safeguard the health, welfare and safety of people, animals, and their shared environment.

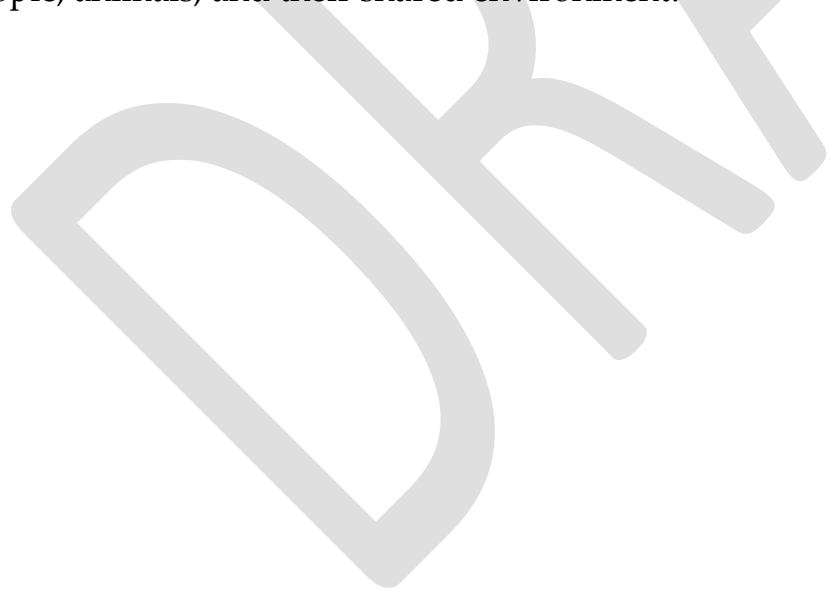




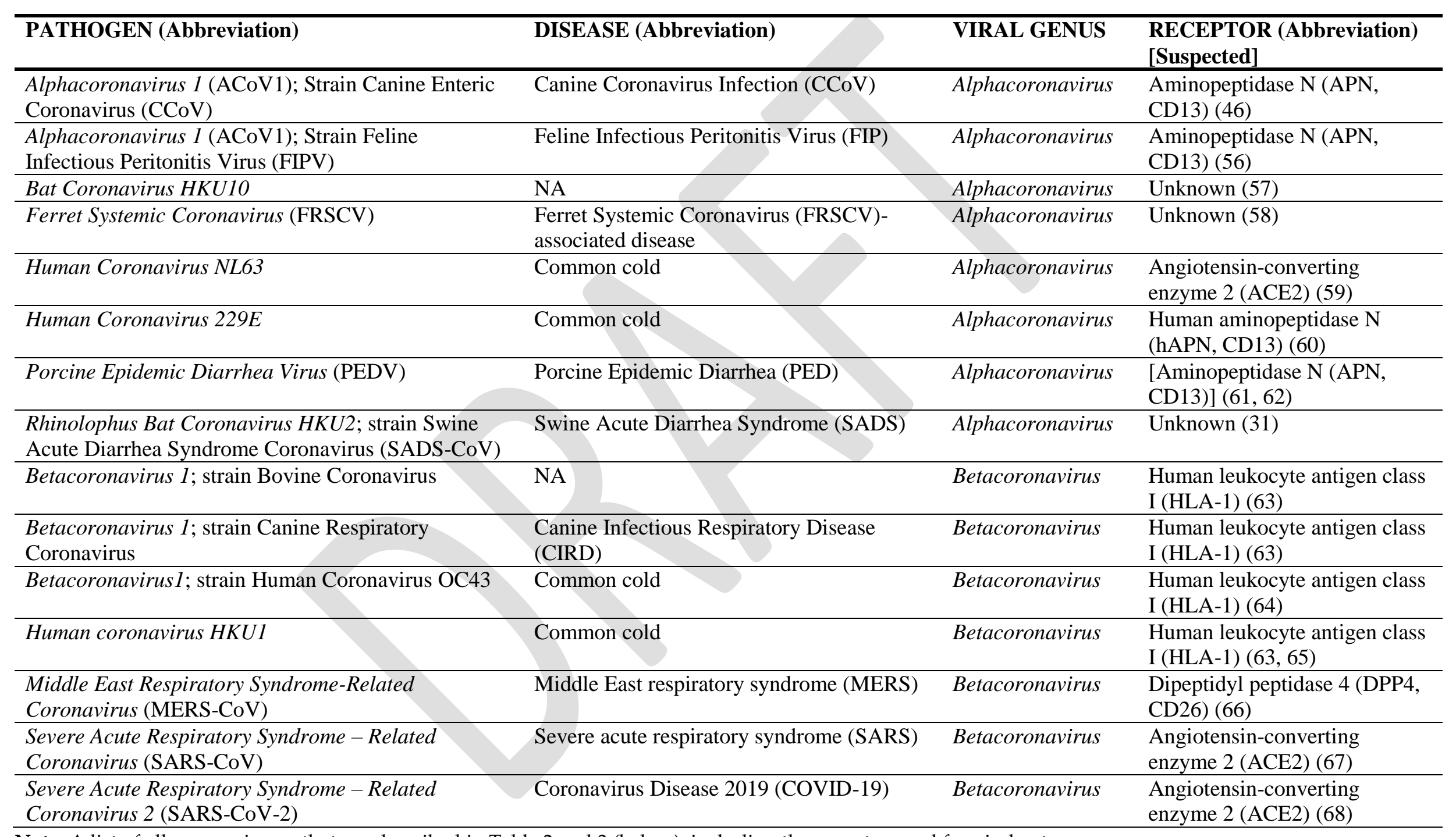

3 Note: A list of all coronaviruses that are described in Table 2 and 3 (below), including the receptor used for viral entry. 
Table 2. - Hosts and reservoirs of current or previously emerging coronaviruses.

\begin{tabular}{|c|c|c|c|c|c|}
\hline PATHOGEN & $\begin{array}{l}\text { RESERVOIR HOST(S) } \\
\text { [Suspected] }\end{array}$ & $\begin{array}{l}\text { INTERMEDIATE / } \\
\text { AMPLIFYING HOST(S) } \\
\text { [Suspected] }\end{array}$ & SPILLOVER HOST(S) & $\begin{array}{l}\text { SUSCEPTIBLE } \\
\text { HOST(S) [Suspected] }\end{array}$ & $\begin{array}{l}\text { NON- } \\
\text { SUSCEPTIBLE } \\
\text { HOST(S) } \\
\end{array}$ \\
\hline $\begin{array}{l}\text { Alphacoronavirus } 1 \\
\text { (ACoV1); Strain Canine } \\
\text { Enteric Coronavirus } \\
(\mathrm{CCoV})\end{array}$ & $\begin{array}{l}\text { Domestic dog } \\
\text { (Canis lupus } \\
\text { familiaris) }\end{array}$ & Unknown/None identified & $\begin{array}{l}\text { Carnivores; } \\
\text { family Canidae } \\
\text { Carnivores; } \\
\text { family Felidae } \\
\text { Carnivores; } \\
\text { family Mustelidae } \\
\text { Carnivores; } \\
\text { family Viverridae }\end{array}$ & Unknown/None identified & $\begin{array}{l}\text { Unknown/None } \\
\text { identified }\end{array}$ \\
\hline $\begin{array}{l}\text { Alphacoronavirus1 } \\
\text { (ACoV1); Strain Feline } \\
\text { Infectious Peritonitis Virus } \\
\text { (FIPV) }\end{array}$ & $\begin{array}{l}\text { Domestic cat } \\
\text { (Felis catus) }\end{array}$ & Unknown/None identified & $\begin{array}{l}\text { Carnivores: } \\
\text { family Felidae }\end{array}$ & $\begin{array}{l}\text { Mouse } \\
\text { (Mus musculus) }\end{array}$ & \\
\hline Bat Coronavirus HKU10 & $\begin{array}{l}\text { Leschenault's } \\
\text { rousettes bat } \\
\text { (Rousettus } \\
\text { leschenaulti) }\end{array}$ & Unknown/None identified & $\begin{array}{l}\text { Pomona leaf- } \\
\text { nosed bats } \\
\text { (Hipposideros } \\
\text { pomona) } \\
\end{array}$ & Unknown/None identified & $\begin{array}{l}\text { Unknown/None } \\
\text { identified }\end{array}$ \\
\hline $\begin{array}{l}\text { Ferret Systemic } \\
\text { Coronavirus (FRSCV) }\end{array}$ & $\begin{array}{l}\text { Ferret } \\
\text { (Mustela putorius) }\end{array}$ & Unknown/None identified & $\begin{array}{l}\text { Ferret } \\
\text { (Mustela putorius) }\end{array}$ & Unknown/None identified & $\begin{array}{l}\text { Unknown/None } \\
\text { identified }\end{array}$ \\
\hline Human coronavirus NL63 & $\begin{array}{l}\text { Bats; family } \\
\text { Hipposideridae }\end{array}$ & Unknown/None identified & Human & Unknown/None identified & $\begin{array}{l}\text { Cattle (Bos } \\
\text { taurus) } \\
\text { Domestic pig } \\
\text { (Sus scrofa) } \\
\text { Donkey } \\
\text { (Equus } \\
\text { africanus) } \\
\text { Goat } \\
\text { (Capra } \\
\text { aegagrus) } \\
\text { Sheep } \\
\text { (Ovis aries) }\end{array}$ \\
\hline Human Coronavirus $229 E$ & $\begin{array}{l}\text { Bats; family } \\
\text { Hipposideridae }\end{array}$ & $\begin{array}{l}\text { Dromedary camels } \\
\text { (Camelus dromedarius) }\end{array}$ & $\begin{array}{l}\text { Alpaca } \\
\text { (Vicugna pacos) }\end{array}$ & $\begin{array}{l}\text { Domestic cat } \\
\text { (Felis catus) }\end{array}$ & \\
\hline
\end{tabular}




\begin{tabular}{|c|c|c|c|c|c|c|c|c|c|}
\hline $\begin{array}{l}\text { Porcine Epidemic } \\
\text { Diarrhea Virus (PEDV) }\end{array}$ & $\begin{array}{l}\text { Bats; family } \\
\text { Vespertillionidae }\end{array}$ & $\begin{array}{l}(92, \\
93)\end{array}$ & Unknown/None identified & $\begin{array}{l}\text { Domestic pig } \\
\text { (Sus scrofa) }\end{array}$ & (30) & \multicolumn{2}{|c|}{ Unknown/None identified } & \multicolumn{2}{|l|}{$\begin{array}{l}\text { Unknown/None } \\
\text { identified }\end{array}$} \\
\hline $\begin{array}{l}\text { Rhinolophus Bat } \\
\text { Coronavirus HKU2; Strain } \\
\text { Swine Acute Diarrhea } \\
\text { Syndrome Coronavirus } \\
\text { (SADS-CoV) }\end{array}$ & $\begin{array}{l}\text { Bats; family } \\
\text { Rhinolophidae }\end{array}$ & (31) & Unknown/None identified & $\begin{array}{l}\text { Domestic pig } \\
\text { (Sus scrofa) }\end{array}$ & (31) & $\begin{array}{l}\text { Mouse } \\
\text { (Mus musculus) }\end{array}$ & (94) & $\begin{array}{l}\text { Unknown/Non } \\
\text { identified }\end{array}$ & \\
\hline $\begin{array}{l}\text { Betacoronavirus } 1 ; \text { strain } \\
\text { Bovine Coronavirus }\end{array}$ & $\begin{array}{l}\text { Rodent; family } \\
\text { Muridae }\end{array}$ & (95) & Unknown/None identified & $\begin{array}{l}\text { Cattle } \\
\text { (Bos taurus) }\end{array}$ & (96) & $\begin{array}{l}\text { Turkey } \\
\text { (Meleagris gallopavo) }\end{array}$ & (97) & $\begin{array}{l}\text { Chicken } \\
\text { (Gallus gallus) }\end{array}$ & (97) \\
\hline $\begin{array}{l}\text { Betacoronavirus } 1 \text {; strain } \\
\text { Canine Respiratory } \\
\text { coronavirus }\end{array}$ & $\begin{array}{l}\text { Rodent; family } \\
\text { Muridae }\end{array}$ & (95) & $\begin{array}{l}{[\text { Cattle }]} \\
\text { (Bos taurus) }\end{array}$ & $\begin{array}{l}\text { Domestic dog } \\
\text { (Canis lupus } \\
\text { familiaris) }\end{array}$ & $(42)$ & Unknown/None id & tified & $\begin{array}{l}\text { Unknown/Non } \\
\text { identified }\end{array}$ & \\
\hline $\begin{array}{l}\text { Betacoronavirus } 1 ; \text { strain } \\
\text { Human coronavirus OC43 }\end{array}$ & $\begin{array}{l}\text { Rodent; family } \\
\text { Muridae }\end{array}$ & (95) & $\begin{array}{l}\text { Artiodactyl; family } \\
\text { Bovidae }\end{array}$ & $\begin{array}{l}\text { Chimpanzees } \\
\text { (Pan troglodytes) }\end{array}$ & (98) & $\begin{array}{l}\text { Mouse } \\
\text { (Mus musculus) }\end{array}$ & (99) & $\begin{array}{l}\text { Unknown/Non } \\
\text { identified }\end{array}$ & \\
\hline Human coronavirus HKUI & $\begin{array}{l}\text { Rodent; family } \\
\text { Muridae }\end{array}$ & $\begin{array}{l}(20, \\
100)\end{array}$ & Unknown/None identified & Human & (9) & Unknown/None id & tified & $\begin{array}{l}\text { Unknown/Non } \\
\text { identified }\end{array}$ & \\
\hline $\begin{array}{l}\text { Middle East Respiratory } \\
\text { Syndrome-Related } \\
\text { Coronavirus (MERS-CoV) }\end{array}$ & $\begin{array}{l}\text { Bats; family } \\
\text { Vespertillionidae }\end{array}$ & $\begin{array}{l}(100, \\
101)\end{array}$ & $\begin{array}{l}\text { Dromedary camels } \\
\text { (Camelus dromedarius) }\end{array}$ & Human & (10) & $\begin{array}{l}\begin{array}{l}\text { Alpaca } \\
\text { (Vicugna pacos) }\end{array} \\
\text { Common } \\
\text { marmoset } \\
\text { (Callithrix jacchus) } \\
\begin{array}{l}\text { Domestic pig } \\
\text { (Sus scrofa) }\end{array} \\
\text { Llama } \\
\text { (Lama glama) } \\
\text { Rhesus macaque } \\
\text { (Macaca mulatta) } \\
\text { New Zealand } \\
\text { White rabbit }\end{array}$ & $\begin{array}{l}(103) \\
(105, \\
106) \\
(108) \\
(108) \\
(110, \\
111) \\
(112)\end{array}$ & 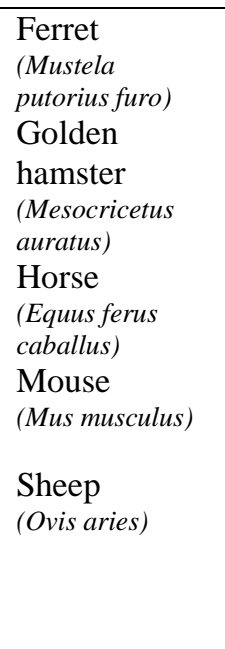 & $\begin{array}{l}(108) \\
(109) \\
(108)\end{array}$ \\
\hline
\end{tabular}


(Oryctolagus

cunuculus)

\begin{tabular}{|c|c|c|c|c|c|c|c|c|c|c|}
\hline \multirow[t]{10}{*}{$\begin{array}{l}\text { Severe Acute Respiratory } \\
\text { Syndrome - Related } \\
\text { Coronavirus (SARS-CoV) }\end{array}$} & \multirow[t]{10}{*}{$\begin{array}{l}\text { Bats; family } \\
\text { Rhinolophidae }\end{array}$} & \multirow[t]{10}{*}{$\begin{array}{l}(57, \\
113- \\
115)\end{array}$} & \multirow{10}{*}{\multicolumn{2}{|c|}{$\begin{array}{l}\text { Masked palm civet } \\
\text { (Paguma larvata) }\end{array}$}} & \multirow{2}{*}{$\begin{array}{l}\text { Chinese ferret } \\
\text { badger } \\
\text { (Melogale moschata) }\end{array}$} & $\begin{array}{l}(21, \\
114)\end{array}$ & \multirow{2}{*}{$\begin{array}{l}\text { African green } \\
\text { monkey } \\
\text { (Chlorocebus } \\
\text { aethiops) } \\
\text { Cynomolgus } \\
\text { macaque } \\
\text { (Macaca fascicularis) }\end{array}$} & (114) & \multirow[t]{10}{*}{$\begin{array}{l}\text { Chickens } \\
\text { (Gallus gallus) }\end{array}$} & \multirow[t]{10}{*}{$(117)$} \\
\hline & & & & & & $\begin{array}{l}(50, \\
114 \\
118)\end{array}$ & & $\begin{array}{l}(114, \\
119)\end{array}$ & & \\
\hline & & & & & $\begin{array}{l}\text { Domestic dog } \\
\text { (Canis lupus } \\
\text { familiaris) } \\
\text { Domestic pig } \\
\text { (Sus scrofa) }\end{array}$ & $\begin{array}{l}(35, \\
114)\end{array}$ & $\begin{array}{l}\text { Domestic cat } \\
\text { (Felis catus) } \\
\text { Domestic pig } \\
\text { (Sus scrofa) }\end{array}$ & $\begin{array}{l}(50, \\
114) \\
(114, \\
117)\end{array}$ & & \\
\hline & & & & & Human & $\begin{array}{l}(120, \\
121)\end{array}$ & $\begin{array}{l}\text { Ferret } \\
\text { (Mustela putorius) }\end{array}$ & $\begin{array}{l}(50, \\
114)\end{array}$ & & \\
\hline & & & & & $\begin{array}{l}\text { Racoon dog } \\
\text { (Nyctereutes }\end{array}$ & $\begin{array}{l}(21, \\
114)\end{array}$ & $\begin{array}{l}\text { Golden hamster } \\
\text { (Mesocricetus auratus) }\end{array}$ & $\begin{array}{l}(114, \\
122)\end{array}$ & & \\
\hline & & & & & & & $\begin{array}{l}\text { Guinea pig } \\
\text { (Cavia porcellus) }\end{array}$ & $(114)$ & & \\
\hline & & & & & & & $\begin{array}{l}\text { Masked palm } \\
\text { civet } \\
\text { (Paguma larvata) }\end{array}$ & $\begin{array}{l}(114, \\
123)\end{array}$ & & \\
\hline & & & & & & & $\begin{array}{l}\text { Mouse } \\
\text { (Mus musculus) }\end{array}$ & $\begin{array}{l}(114, \\
124)\end{array}$ & & \\
\hline & & & & & & & $\begin{array}{l}\text { Rat } \\
\text { (Rattus sp) }\end{array}$ & (114) & & \\
\hline & & & & & & & $\begin{array}{l}\text { Rhesus macaque } \\
\text { (Macaca mulatta) }\end{array}$ & $\begin{array}{l}(114, \\
125)\end{array}$ & & \\
\hline \multirow{3}{*}{$\begin{array}{l}\text { Severe Acute Respiratory } \\
\text { Syndrome - Related } \\
\text { Coronavirus } 2 \text { (SARS- } \\
\text { CoV-2) }\end{array}$} & $\begin{array}{l}\text { [Bats: family } \\
\text { Rhinolophidae] }\end{array}$ & (3) & \multirow{3}{*}{\multicolumn{2}{|c|}{ Unknown/None identified }} & $\begin{array}{l}\text { Domestic cat } \\
\text { (Felis catus) }\end{array}$ & $\begin{array}{l}(14, \\
26)\end{array}$ & $\begin{array}{l}\text { Cynomolgus } \\
\text { macaque }\end{array}$ & $(126)$ & $\begin{array}{l}\text { Chickens } \\
\text { (Gallus gallus) }\end{array}$ & $\begin{array}{l}(27, \\
28)\end{array}$ \\
\hline & & & & & $\begin{array}{l}\text { Domestic dog } \\
\text { (Canis lupus } \\
\text { familiaris) }\end{array}$ & $(51)$ & $\begin{array}{l}\text { Domestic cat } \\
\text { (Felis catus) }\end{array}$ & $\begin{array}{l}(27, \\
54)\end{array}$ & $\begin{array}{l}\text { Domestic pig } \\
\text { (Sus scrofa) }\end{array}$ & $\begin{array}{l}(27, \\
28)\end{array}$ \\
\hline & & & & & Human & (127) & $\begin{array}{l}\text { Domestic dog } \\
\text { (Canis lupus } \\
\text { familiaris) }\end{array}$ & (27) & $\begin{array}{l}\text { Japanese } \\
\text { Quail }\end{array}$ & $\begin{array}{l}\text { (pers. } \\
\text { comm) }\end{array}$ \\
\hline
\end{tabular}




\begin{tabular}{|c|c|c|c|c|c|}
\hline Mink & $\begin{array}{l}(26, \\
36)\end{array}$ & $\begin{array}{l}\text { Egyptian fruit bat } \\
\text { (Rousettus }\end{array}$ & (28) & $\begin{array}{l}\text { (Coturnix } \\
\text { japonica) } \\
\text { Mouse } \\
\text { (Mus musculus) }\end{array}$ & (128) \\
\hline $\begin{array}{l}\text { Lion } \\
\text { (Panthera leo) }\end{array}$ & (26) & $\begin{array}{l}\text { aegyptiacus) } \\
\text { Ferret } \\
\text { (Mustela putorius) }\end{array}$ & $\begin{array}{l}(27, \\
28, \\
53)\end{array}$ & $\begin{array}{l}\text { Pekin duck } \\
\text { (Anas } \\
\text { platyrhinchos) }\end{array}$ & $\begin{array}{l}\text { (pers. } \\
\text { comm) }\end{array}$ \\
\hline $\begin{array}{l}\text { Puma } \\
\text { (Puma col }\end{array}$ & (26) & $\begin{array}{l}\text { Golden hamster } \\
\text { (Mesocricetus auratus }\end{array}$ & (52) & $\begin{array}{l}\text { Turkey } \\
\text { (Meleagris } \\
\text { gallopavo) }\end{array}$ & $\begin{array}{l}\text { (pers. } \\
\text { comm) }\end{array}$ \\
\hline $\begin{array}{l}\text { Tiger } \\
\text { (Panthera tigris) }\end{array}$ & (26) & $\begin{array}{l}\text { Rhesus macaque } \\
\text { (Macaca mulatta) }\end{array}$ & (129) & $\begin{array}{l}\text { White } \\
\text { Chinese } \\
\text { geese (Anser } \\
\text { cygnoides) }\end{array}$ & $\begin{array}{l}\text { (pers. } \\
\text { comm) }\end{array}$ \\
\hline
\end{tabular}

Note: All hosts discovered for each coronavirus are presented below. For the purposes of this table: Reservoir host - a species in which the pathogen endemically circulates, and is considered to have co-evolved with; Intermediate host - species that harbor a recent common ancestor of the coronavirus and/or played a role in the natural selection/adaptation of the virus before its spillover. Spillover host - non-endemically infected species where one or more individuals are considered to have acquired the virus through natural infection (e.g., exposure an infected conspecific or inter-species transmission). Susceptible hosts - species where one or more individuals can become infected with the virus through experimental challenges or otherwise laboratorycontrolled infection studies. In vitro studies, or studies using transgenic animal models to induce susceptibility were not included in this table. Non-susceptible hosts - species where experimental challenge or otherwise laboratory-controlled infection studies have occurred that did not result in viral infection. For all categories, infection is considered detection of viral RNA from host samples and/or the host mounting a detectable antibody response. Therefore, infection as it is used here does not necessarily imply hosts naturally or experimentally infected with a virus are capable of transmitting the infection to others.

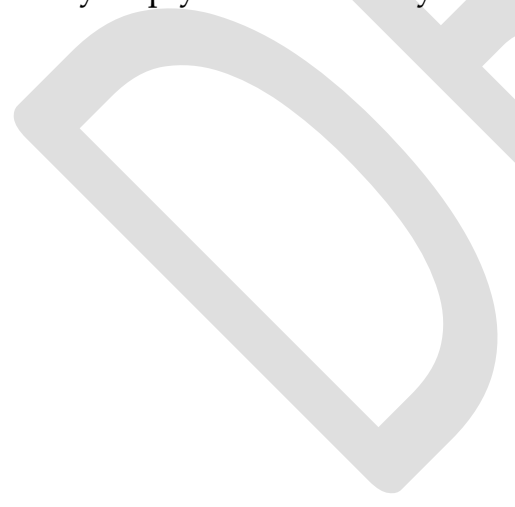


Table 3. - Clinical presentation of current or previously emerging coronaviruses.

\begin{tabular}{|c|c|c|c|c|c|c|c|}
\hline \multirow{6}{*}{$\begin{array}{l}\text { PATHOGEN } \\
\text { Alphacoronavirus } 1 \\
\text { (ACoV1); Strain } \\
\text { Canine Enteric } \\
\text { Coronavirus }(\mathrm{CCoV})\end{array}$} & \multirow{2}{*}{$\frac{\text { HOST }}{\text { Domestic dog }}$} & \multirow{2}{*}{$\begin{array}{l}\text { HOST TYPE } \\
\text { Reservoir }\end{array}$} & \multirow{2}{*}{$\begin{array}{l}\text { BODY SYSTEM } \\
\text { Gastrointestinal }\end{array}$} & \multicolumn{2}{|l|}{ CLINICAL PRESENTATION } & \multicolumn{2}{|c|}{ VACCINE } \\
\hline & & & & Vomiting, diarrhea, and dehydration & $\begin{array}{l}(130) \\
(49)\end{array}$ & Yes & $(130)$ \\
\hline & $\begin{array}{l}\text { Carnivores; family } \\
\text { Canidae }\end{array}$ & Spillover & Unknown & Unknown & $\begin{array}{l}(70) \\
(72)\end{array}$ & No & $(131)$ \\
\hline & $\begin{array}{l}\text { Carnivores; } \\
\text { family Felidae }\end{array}$ & Spillover & Gastrointestinal & Mild diarrhea & (73) & No & $(131)$ \\
\hline & $\begin{array}{l}\text { Carnivores; family } \\
\text { Mustelidae }\end{array}$ & Spillover & Unknown & Unknown & $(71)$ & No & $(131)$ \\
\hline & $\begin{array}{l}\text { Carnivores; family } \\
\text { Viverridae }\end{array}$ & Susceptible & Unknown & Unknown & $(71)$ & No & $(131)$ \\
\hline $\begin{array}{l}\text { Alphacoronavirus } 1 \\
\text { (ACoV1); Strain } \\
\text { Feline Infectious }\end{array}$ & Domestic cat & Reservoir & & $\begin{array}{l}\text { Varies: fever, weight loss, diarrhea, ascites, } \\
\text { thoracic effusion, ocular signs, neurologic signs, } \\
\text { and death }\end{array}$ & $\begin{array}{l}(130, \\
132)\end{array}$ & Yes* & $(130)$ \\
\hline $\begin{array}{l}\text { Peritonitis Virus } \\
\text { (FIPV) }\end{array}$ & $\begin{array}{l}\text { Carnivores: family } \\
\text { Felidae }\end{array}$ & Spillover & Multi-systemic & Weight loss, fever, diarrhea, jaundice & $(133)$ & No & $(131)$ \\
\hline \multirow[t]{2}{*}{$\begin{array}{l}\text { Bat Coronavirus } \\
\text { HKU10 }\end{array}$} & $\begin{array}{l}\text { Leschenault's rousettes } \\
\text { bat }\end{array}$ & Reservoir & NA & Asymptomatic & $(81)$ & No & $(131)$ \\
\hline & Pomona leaf-nosed bats & Spillover & Unknown & Lower body weight than non-infected bats & $(81)$ & No & $(131)$ \\
\hline $\begin{array}{l}\text { Ferret Systemic } \\
\text { Coronavirus (FRSCV) }\end{array}$ & Ferret & $\begin{array}{l}\text { Reservoir, } \\
\text { Spillover }\end{array}$ & Multi-systemic & $\begin{array}{l}\text { Anorexia, weight loss, diarrhea, } \\
\text { lymphadenopathy, anemia, and neurologic signs }\end{array}$ & (134) & No & (131) \\
\hline \multirow[t]{2}{*}{$\begin{array}{l}\text { Human coronavirus } \\
\text { NL63 }\end{array}$} & $\begin{array}{l}\text { Bats; family } \\
\text { Hipposideridae }\end{array}$ & Reservoir & NA & Asymptomatic & (93) & No & $(131)$ \\
\hline & Human & Spillover & Respiratory & Fever, cough, rhinorrhea & $(135)$ & No & $(136)$ \\
\hline \multirow[t]{5}{*}{$\begin{array}{l}\text { Human Coronavirus } \\
229 E\end{array}$} & $\begin{array}{l}\text { Bats; family } \\
\text { Hipposideridae }\end{array}$ & & NA & Asymptomatic & $(93)$ & No & $(131)$ \\
\hline & Dromedary camels & Intermediate & NA & Asymptomatic & $(34)$ & No & $(131)$ \\
\hline & Alpaca & Spillover & Respiratory & Acute respiratory signs, death & $(137)$ & No & $(131)$ \\
\hline & Human & Spillover & Respiratory & $\begin{array}{l}\text { Nasal congestion, rhinorrhea. Can progress to } \\
\text { pneumonia. }\end{array}$ & $(138)$ & No & $(136)$ \\
\hline & Domestic cat & Susceptible & NA & Asymptomatic & $(91)$ & No & $(131)$ \\
\hline \multirow{2}{*}{$\begin{array}{l}\text { Porcine Epidemic } \\
\text { Diarrhea Virus } \\
\text { (PEDV) }\end{array}$} & $\begin{array}{l}\text { Bats; family } \\
\text { Vespertillionidae }\end{array}$ & Reservoir & Unknown & Unknown & (93) & No & $(131)$ \\
\hline & Domestic pig & Spillover & Gastrointestinal & $\begin{array}{l}\text { Vomiting, diarrhea, and death in piglets, } \\
\text { diarrhea and agalactia in pregnant sows }\end{array}$ & $\begin{array}{l}(130, \\
139)\end{array}$ & $\mathrm{Yes}^{+}$ & $(131)$ \\
\hline
\end{tabular}




\begin{tabular}{|c|c|c|c|c|c|c|c|}
\hline Rhinolophus Bat & Bats; family & Reservoir & NA & Asymptomatic & (93) & No & (131) \\
\hline $\begin{array}{l}\text { Coronavirus HKU2; } \\
\text { strain Swine Acute } \\
\text { Diarrhea Syndrome }\end{array}$ & $\begin{array}{l}\text { Rhinolophidae } \\
\text { Domestic pig }\end{array}$ & Spillover & Gastrointestinal & $\begin{array}{l}\text { Severe, acute diarrhea, rapid weight loss, death } \\
\text { in piglets }\end{array}$ & (140) & No & (131) \\
\hline $\begin{array}{l}\text { Coronavirus (SADS- } \\
\mathrm{CoV} \text { ) }\end{array}$ & Mouse & Susceptible & NA & Asymptomatic & (94) & NA & (94) \\
\hline \multirow{3}{*}{$\begin{array}{l}\text { Betacoronavirus } 1 ; \\
\text { strain Bovine } \\
\text { Coronavirus }\end{array}$} & Rodent; family Muridae & Reservoir & Unknown, & Unknown & (95) & No & (131) \\
\hline & Cattle & Spillover & $\begin{array}{l}\text { Respiratory, } \\
\text { gastrointestinal }\end{array}$ & Respiratory signs, diarrhea, anorexia, lethargy & (130) & Yes & (130) \\
\hline & Turkey & Susceptible & Gastrointestinal & Diarrhea, reduced weight gain, and enteritis & (97) & NA & \\
\hline \multirow{3}{*}{$\begin{array}{l}\text { Betacoronavirus 1; } \\
\text { strain Canine } \\
\text { Respiratory } \\
\text { coronavirus }\end{array}$} & Rodent; family Muridae & Reservoir & Unknown & Unknown & (95) & No & (131) \\
\hline & [Cattle] & Intermediate & Unknown & Unknown & (43) & No & (130) \\
\hline & Domestic dog & Spillover & Respiratory & $\begin{array}{l}\text { Mild cough, nasal discharge, inappetence, } \\
\text { bronchopneumonia }\end{array}$ & (43) & No & (42) \\
\hline \multirow{5}{*}{$\begin{array}{l}\text { Betacoronavirus1; } \\
\text { strain Human } \\
\text { coronavirus OC43 }\end{array}$} & Rodent; family Muridae & Reservoir & Unknown, & Unknown & (95) & No & (131) \\
\hline & $\begin{array}{l}\text { Artiodactyl; family } \\
\text { Bovidae }\end{array}$ & Intermediate & $\begin{array}{l}\text { Unknown, } \\
\text { suspected host }\end{array}$ & Unknown & (43) & No & (131) \\
\hline & Chimpanzees & Spillover & Respiratory & Coughing, sneezing & (25) & No & (131) \\
\hline & Human & Spillover & Respiratory & $\begin{array}{l}\text { Nasal congestion, rhinorrhea. Can progress to } \\
\text { pneumonia }\end{array}$ & (6) & No & (136) \\
\hline & Mouse & Susceptible & Neurological & Anorexia, weight loss, neurologic signs & (99) & No & (131) \\
\hline \multirow[t]{2}{*}{$\begin{array}{l}\text { Human coronavirus } \\
\text { HKU1 }\end{array}$} & Rodent; family Muridae & Reservoir & $\begin{array}{l}\text { Unknown, } \\
\text { suspected host }\end{array}$ & Unknown, suspected host & (20) & No & (131) \\
\hline & Human & Spillover & Respiratory & Rhinorrhea, cough, fever & (141) & No & (136) \\
\hline \multirow{9}{*}{$\begin{array}{l}\text { Middle East } \\
\text { Respiratory } \\
\text { Syndrome-Related } \\
\text { Coronavirus (MERS- } \\
\text { CoV) }\end{array}$} & Bats; family & Reservoir & $\mathrm{NA}$ & Asymptomatic & $(93)$ & No & $(131)$ \\
\hline & Vespertillionidae & & & & & & \\
\hline & Dromedary camels & Intermediate & Respiratory & $\begin{array}{l}\text { Often asymptomatic, or can present with fever, } \\
\text { nasal discharge }\end{array}$ & (142) & No & (131) \\
\hline & Human & Spillover & $\begin{array}{l}\text { Respiratory, multi- } \\
\text { systemic }\end{array}$ & $\begin{array}{l}\text { Variable: coughing, shortness of breath, fatigue, } \\
\text { myalgia, arthralgia, fever, headaches, vomiting, } \\
\text { and diarrhea, sore throat and rhinorrhea. Can } \\
\text { progress to pneumonia, acute renal failure, } \\
\text { multiorgan failure, and death }\end{array}$ & (142) & No & (143) \\
\hline & Alpaca & Susceptible & NA & Asymptomatic & (103) & No & (131) \\
\hline & Common marmoset & Susceptible & Respiratory & Pneumonia & (105) & No & (131) \\
\hline & Domestic pig & Susceptible & Respiratory & Nasal discharge & (108) & No & (131) \\
\hline & Llama & Susceptible & Respiratory & Nasal discharge & (108) & No & (131) \\
\hline & Rhesus macaque & Susceptible & $\mathrm{NA}$ & Asymptomatic & (112) & No & (131) \\
\hline
\end{tabular}




\begin{tabular}{|c|c|c|c|c|c|c|c|}
\hline & $\begin{array}{l}\text { New Zealand White } \\
\text { rabbit }\end{array}$ & Susceptible & Respiratory & Asymptomatic & (112) & No & (131) \\
\hline \multirow{16}{*}{$\begin{array}{l}\text { Severe Acute } \\
\text { Respiratory Syndrome } \\
\text { - Related Coronavirus } \\
\text { (SARS-CoV) }\end{array}$} & $\begin{array}{l}\text { Bats; family } \\
\text { Rhinolonhidae }\end{array}$ & Reservoir & Unknown, & Unknown & (93) & No & (131) \\
\hline & Masked palm civet & $\begin{array}{l}\text { Intermediate, } \\
\text { Susceptible }\end{array}$ & Respiratory & Fever, lethargy, pneumonia & $\begin{array}{l}(114, \\
123)\end{array}$ & No & (131) \\
\hline & Chinese ferret badger & Spillover & NA & Asymptomatic & (114) & No & (131) \\
\hline & Domestic cat & $\begin{array}{l}\text { Spillover, } \\
\text { Susceptible }\end{array}$ & NA & Asymptomatic & $\begin{array}{l}(50, \\
114)\end{array}$ & No & (131) \\
\hline & Domestic dog & Spillover & Unknown & Unknown & (118) & No & (131) \\
\hline & Domestic pig & $\begin{array}{l}\text { Spillover, } \\
\text { Susceptible }\end{array}$ & NA & Asymptomatic & $\begin{array}{l}(114, \\
117)\end{array}$ & No & (131) \\
\hline & Human & Spillover & Respiratory & $\begin{array}{l}\text { Fever, cough, shortness of breath, difficulty } \\
\text { breathing, pneumonia }\end{array}$ & (132) & No & (143) \\
\hline & Racoon dog & Spillover & NA & Asymptomatic & $\begin{array}{l}(114, \\
123)\end{array}$ & No & (131) \\
\hline & African green monkey & Susceptible & NA & Asymptomatic & (114) & No & (131) \\
\hline & Cynomolgus macaque & Susceptible & Respiratory & Lethargy, skin rash, and respiratory distress & $\begin{array}{l}(114, \\
119)\end{array}$ & No & (131) \\
\hline & Ferret & Susceptible & Respiratory & Lethargy, conjunctivitis, weight loss & $\begin{array}{l}(50, \\
114)\end{array}$ & No & (131) \\
\hline & Golden hamster & Susceptible & NA & Asymptomatic & $\begin{array}{l}(114, \\
122)\end{array}$ & No & (131) \\
\hline & Guinea pig & Susceptible & NA & Asymptomatic & (114) & No & (131) \\
\hline & Mouse & Susceptible & Non-specific & Asymptomatic or weight loss and dehydration & $\begin{array}{l}(114, \\
124)\end{array}$ & No & (131) \\
\hline & & Susceptible & NA & Asymptomatic & (114) & No & (131) \\
\hline & Rhesus macaque & Susceptible & Non-specific & Transient fever & $\begin{array}{l}(114, \\
125)\end{array}$ & No & (131) \\
\hline \multirow{4}{*}{$\begin{array}{l}\text { Severe Acute } \\
\text { Respiratory Syndrome } \\
\text { - Related Coronavirus } \\
2 \text { (SARS-CoV-2) }\end{array}$} & $\begin{array}{l}\text { [Bats: family } \\
\text { Rhinolophidae] }\end{array}$ & Reservoir & Unknown & Unknown & (3) & No & NA \\
\hline & Domestic cat & $\begin{array}{l}\text { Spillover, } \\
\text { Susceptible }\end{array}$ & Respiratory & $\begin{array}{l}\text { Asymptomatic or ocular discharge and sneezing, } \\
\text { or death in juvenile cats }\end{array}$ & $\begin{array}{l}(14, \\
26, \\
27)\end{array}$ & No & NA \\
\hline & Domestic dog & $\begin{array}{l}\text { Spillover, } \\
\text { Susceptible }\end{array}$ & NA & Asymptomatic & (51) & No & NA \\
\hline & Human & Spillover & Respiratory & $\begin{array}{l}\text { Fever, cough, shortness of breath or difficulty } \\
\text { breathing }\end{array}$ & & No & NA \\
\hline
\end{tabular}




\begin{tabular}{|c|c|c|c|c|c|c|}
\hline Mink & Spillover & $\begin{array}{l}\text { Gastrointestinal } \\
\text { and Respiratory }\end{array}$ & $\begin{array}{l}\text { Respiratory and gastrointestinal signs, increased } \\
\text { mortality }\end{array}$ & $\begin{array}{l}(26, \\
36 \\
144)\end{array}$ & No & NA \\
\hline Lion & Spillover & Respiratory & Cough, inappetence, wheezing & (26) & No & NA \\
\hline Puma & Spillover & Unknown & Unknown & (26) & No & NA \\
\hline Tiger & Spillover & Respiratory & Cough, inappetence, wheezing & (26) & No & NA \\
\hline Cynomolgus macaque & Susceptible & Respiratory & Asymptomatic, nasal discharge & (126) & No & NA \\
\hline Egyptian fruit bat & Susceptible & & Asymptomatic, mild rhinitis & $(28)$ & & NA \\
\hline Ferret & Susceptible & Respiratory & Asymptomatic or fever, cough, inappetence & $\begin{array}{l}(27, \\
28, \\
53)\end{array}$ & No & NA \\
\hline Golden hamster & Susceptible & Respiratory & $\begin{array}{l}\text { Weight loss, lethargy, ruffled fur, hunched } \\
\text { posture, tachypnea }\end{array}$ & (52) & No & NA \\
\hline Rhesus macaque & Susceptible & Respiratory & $\begin{array}{l}\text { Changes in respiratory pattern, piloerection, } \\
\text { reduced appetite, pale appearance, hunched } \\
\text { posture, dehydration, coughing }\end{array}$ & (129) & No & NA \\
\hline
\end{tabular}

27 Note: The clinical presentation of disease in all hosts described in Tables 1 and 2 (above) other than non-susceptible hosts. ${ }^{*}=$ Not recommended by the AAFP 28 for administration; ${ }^{+}$Conditional license for use. 


\section{Reference:}

30 1. Woo PCY, Lau SKP, Huang Y, Yuen K-Y. Coronavirus diversity, phylogeny and interspecies 31 jumping. Experimental Biology and Medicine. 2009;234(10):1117-27.

32 2. Huang C, Wang Y, Li X, Ren L, Zhao J, Hu Y, et al. Clinical features of patients infected with 2019 novel coronavirus in Wuhan, China. The Lancet. 2020;395(10223):497-506.

3. Zhou P, Yang X-L, Wang X-G, Hu B, Zhang L, Zhang W, et al. A pneumonia outbreak associated with a new coronavirus of probable bat origin. Nature. 2020;579(7798):270-3.

4. Drexler JF, Corman VM, Drosten C. Ecology, evolution and classification of bat coronaviruses in the aftermath of SARS. Antiviral Research. 2014;101:45-56.

38 5. Hamre D, Procknow JJ. A new virus isolated from the human respiratory tract. Proceedings of the Society for Experimental Biology and Medicine. 1966;121(1):190-3.

6. McIntosh K, Dees JH, Becker WB, Kapikian AZ, Chanock RM. Recovery in tracheal organ cultures of novel viruses from patients with respiratory disease. Proceedings of the National Academy of Sciences of the United States of America. 1967;57(4):933-40.

7. Vijgen L, Keyaerts E, Moës E, Thoelen I, Wollants E, Lemey P, et al. Complete genomic sequence of human coronavirus OC43: molecular clock analysis suggests a relatively recent zoonotic coronavirus transmission event. Journal of Virology. 2005;79(3):1595.

8. van der Hoek L, Pyrc K, Jebbink MF, Vermeulen-Oost W, Berkhout RJM, Wolthers KC, et al. Identification of a new human coronavirus. Nature Medicine. 2004;10(4):368-73.

9. Woo PCY, Lau SKP, Chu C-m, Chan K-h, Tsoi H-w, Huang Y, et al. Characterization and complete genome sequence of a novel coronavirus, Coronavirus HKU1, from patients with pneumonia. Journal of Virology. 2005;79(2):884.

10. Zaki AM, van Boheemen S, Bestebroer TM, Osterhaus ADME, Fouchier RAM. Isolation of a novel coronavirus from a man with pneumonia in Saudi Arabia. New England Journal of Medicine. 2012;367(19):1814-20.

54 11. Anthony SJ, Johnson CK, Greig DJ, Kramer S, Che X, Wells H, et al. Global patterns in 55 coronavirus diversity. Virus Evolution. 2017;3(1).

56 12. CDC. One Health. 2020 [cited July 15, 2020]; Available from: cdc.gov/onehealth

57 13. Farag E, Nour M, Islam MM, Mustafa A, Khalid M, Sikkema RS, et al. Qatar experience on 58 One Health approach for Middle East respiratory syndrome coronavirus, 2012-2017: A viewpoint. 59 One Health. 2019;7:100090-.

60 14. Newman A, Smith D, Ghai RR, Wallace RM, Torchetti MK, Loiacono C, et al. First reported 61 cases of SARS-CoV-2 infection in companion animals - New York, March-April 2020. Morbidity and 62 Mortality Weekly Report. 2020;69(23):710-3.

63 15. World Health Organization, Food and Agriculture Organization of the United Nations, 64 World Organisation for Animal Health. Taking a multisectoral, One Health approach: a tripartite guide to addressing zoonotic diseases in countries. Geneva: World Health Organization; 2019.

16. Jones KE, Patel NG, Levy MA, Storeygard A, Balk D, Gittleman JL, et al. Global trends in emerging infectious diseases. Nature. 2008;451(7181):990-3.

17. Burgin CJ, Colella JP, Kahn PL, Upham NS. How many species of mammals are there? Journal of Mammalogy. 2018;99(1):1-14.

18. Luis AD, Hayman DTS, O'Shea TJ, Cryan PM, Gilbert AT, Pulliam JRC, et al. A comparison of bats and rodents as reservoirs of zoonotic viruses: are bats special? Proceedings of the Royal Society B: Biological Sciences. 2013;280(1756):20122753.

73 19. Kasso M, Mundanthra B. Ecological and economic importance of bats (Order Chiroptera). 74 International Scholarly Research Notices. 2013;2013:9.

75 20. Corman VM, Muth D, Niemeyer D, Drosten C. Hosts and sources of endemic human 76 coronaviruses. In: Kielian M, Mettenleiter TC, Roossinck MJ, editors. Advances in Virus Research: 77 Academic Press; 2018. p. 163-88. 
21. Guan Y, Zheng BJ, He YQ, Liu XL, Zhuang ZX, Cheung CL, et al. Isolation and characterization of viruses related to the SARS coronavirus from animals in Southern China. Science. 2003;302(5643):276-8.

22. Song H-D, Tu C-C, Zhang G-W, Wang S-Y, Zheng K, Lei L-C, et al. Cross-host evolution of severe acute respiratory syndrome coronavirus in palm civet and human. Proceedings of the National Academy of Sciences. 2005;102(7):2430-5.

23. Köndgen S, Schenk S, Pauli G, Boesch C, Leendertz FH. Noninvasive monitoring of respiratory viruses in wild chimpanzees. EcoHealth. 2010 2010/09/01;7(3):332-41.

24. Scully EJ, Basnet S, Wrangham RW, Muller MN, Otali E, Hyeroba D, et al. Lethal respiratory disease associated with human rhinovirus $C$ in wild chimpanzees, Uganda, 2013. Emerg Infect Dis. 2018;24(2):267-74.

25. Patrono LV, Samuni L, Corman VM, Nourifar L, Röthemeier C, Wittig RM, et al. Human coronavirus OC43 outbreak in wild chimpanzees, Côte d'Ivoire, 2016. Emerging Microbes and Infections. 2018 2018/12/01;7(1):1-4.

26. OIE. COVID 19 Portal Events in Animals. 2020 [cited July 13, 2020]; Available from: https://www.oie.int/en/scientific-expertise/specific-information-and-recommendations/questionsand-answers-on-2019novel-coronavirus/events-in-animals/

27. Shi J, Wen Z, Zhong G, Yang H, Wang C, Huang B, et al. Susceptibility of ferrets, cats, dogs, and other domesticated animals to SARS-coronavirus 2. Science. 2020:eabb7015.

28. Schlottau K, Rissmann M, Graaf A, Schön J, Sehl J, Wylezich C, et al. Experimental transmission studies of SARS-CoV-2 in fruit bats, ferrets, pigs and chickens. The Lancet Microbe. 2020.

29. Wang Q, Vlasova AN, Kenney SP, Saif LJ. Emerging and re-emerging coronaviruses in pigs. Current Opinion in Virology. 2019;34:39-49.

30. Lee C. Porcine epidemic diarrhea virus: an emerging and re-emerging epizootic swine virus. Virology Journal. 2015;12(1):193.

31. Zhou P, Fan H, Lan T, Yang X-L, Shi W-F, Zhang W, et al. Fatal swine acute diarrhoea syndrome caused by an HKU2-related coronavirus of bat origin. Nature. 2018;556(7700):255-8.

32. Amer HM. Bovine-like coronaviruses in domestic and wild ruminants. Animal Health Research Reviews. 2018;19(2):113-24.

33. Vijgen L, Keyaerts E, Lemey P, Maes P, Van Reeth K, Nauwynck H, et al. Evolutionary history of the closely related Group 2 coronaviruses: porcine hemagglutinating encephalomyelitis virus, bovine coronavirus, and human coronavirus OC43. Journal of Virology. 2006;80(14):7270.

34. Corman VM, Eckerle I, Memish ZA, Liljander AM, Dijkman R, Jonsdottir H, et al. Link of a ubiquitous human coronavirus to dromedary camels. Proceedings of the National Academy of Sciences of the United States of America. 2016;113(35):9864-9.

35. Chen W, Yan M, Yang L, Ding B, He B, Wang Y, et al. SARS-associated coronavirus transmitted from human to pig. Emerg Infect Dis. 2005;11(3):446-8.

36. Oreshkova N, Molenaar R-J, Vreman S, Harders F, Munnink BBO, Hakze R, et al. SARS-CoV2 infection in farmed mink, Netherlands, April 2020. Biorxiv. 2020:2020.05.18.101493.

37. CDC. Healthy Pets, Healthy People. 2020 [cited July 13, 2020]; Available from: https://www.cdc.gov/healthypets/index.html

38. AVMA. Pet Ownership and Demographics Sourcebook: American Veterinary Medical Association; 2018.

39. Smith K, Boxrud D, Leano F, Snider C, Braden C, Montgomery S, et al. Outbreak of multidrug-resistant Salmonella typhimurium associated with rodents purchased at retail pet stores --United States, December 2003--October 2004. Morbidity and Mortality Weekly Report. 2005;54(17):429-33.

40. Hampson K, Coudeville L, Lembo T, Sambo M, Kieffer A, Attlan M, et al. Estimating the global burden of endemic canine rabies. PLoS neglected tropical diseases. 2015 Apr;9(4):e0003709.

41. Stull JW, Brophy J, Weese JS. Reducing the risk of pet-associated zoonotic infections. Canadian Medical Association Journal. 2015;187(10):736-43. 
130 42. Erles K, Toomey C, Brooks HW, Brownlie J. Detection of a group 2 coronavirus in dogs with 131 canine infectious respiratory disease. Virology. 2003;310(2):216-23.

132 43. Erles K, Brownlie J. Canine respiratory coronavirus: an emerging pathogen in the canine 133 infectious respiratory disease complex. Veterinary Clinics of North America: Small Animal Practice. 1342008 2008/07/01/;38(4):815-25.

135 44. Erles K, Shiu K-B, Brownlie J. Isolation and sequence analysis of canine respiratory coronavirus. Virus Research. 2007;124(1):78-87.

45. Kaneshima T, Hohdatsu T, Hagino R, Hosoya S, Nojiri Y, Murata M, et al. The infectivity and pathogenicity of a group 2 bovine coronavirus in pups. Journal of Veterinary Medical Science. 2007;69(3):301-3.

46. Licitra BN, Duhamel GE, Whittaker GR. Canine enteric coronaviruses: emerging viral pathogens with distinct recombinant spike proteins. Viruses. 2014;6(8):3363-76.

47. Buonavoglia C, Decaro N, Martella V, Elia G, Campolo M, Desario C, et al. Canine coronavirus highly pathogenic for dogs. Emerg Infect Dis. 2006;12(3):492-4.

48. Barlough JE, Stoddart CA, Sorresso GP, Jacobson RH, Scott FW. Experimental inoculation of cats with canine coronavirus and subsequent challenge with feline infectious peritonitis virus. Laboratory animal science. 1984 Dec;34(6):592-7.

49. Pratelli A. Genetic evolution of canine coronavirus and recent advances in prophylaxis. Veterinary research. 2006 Mar-Apr;37(2):191-200.

50. Martina BEE, Haagmans BL, Kuiken T, Fouchier RAM, Rimmelzwaan GF, van Amerongen G, et al. SARS virus infection of cats and ferrets. Nature. 2003;425(6961):915-.

51. Sit THC, Brackman CJ, Ip SM, Tam KWS, Law PYT, To EMW, et al. Infection of dogs with SARS-CoV-2. Nature. 2020;325.

52. Chan JF-W, Zhang AJ, Yuan S, Poon VK-M, Chan CC-S, Lee AC-Y, et al. Simulation of the clinical and pathological manifestations of Coronavirus Disease 2019 (COVID-19) in golden Syrian hamster model: implications for disease pathogenesis and transmissibility. Clinical Infectious Diseases. 2020.

53. Kim Y-I, Kim S-G, Kim S-M, Kim E-H, Park S-J, Yu K-M, et al. Infection and rapid transmission of SARS-CoV-2 in ferrets. Cell Host \& Microbe. 2020;27(5):704-9.

54. Halfmann PJ, Hatta M, Chiba S, Maemura T, Fan S, Takeda M, et al. Transmission of SARSCoV-2 in domestic cats. New England Journal of Medicine. 2020;Letter to the Editor.

55. Lorusso A, Calistri P, Mercante MT, Monaco F, Portanti O, Marcacci M, et al. A “One-Health" approach for diagnosis and molecular characterization of SARS-CoV-2 in Italy. One Health. 2020;10:100135.

56. Hohdatsu T, Izumiya Y, Yokoyama Y, Kida K, Koyama H. Differences in virus receptor for type I and type II feline infectious peritonitis virus. Archives of virology. 1998 1998/04/01;143(5):83950.

57. Susanna KPL, Patrick CYW, Kenneth SML, Huang Y, Tsoi H-W, Beatrice HLW, et al. Severe acute respiratory syndrome coronavirus-like virus in Chinese horseshoe bats. Proceedings of the National Academy of Sciences of the United States of America. 2005;102(39):14040-5.

58. Murray J, Kiupel M, Maes RK. Ferret coronavirus-associated diseases. Veterinary Clinics: Exotic Animal Practice. 2010;13(3):543-60.

59. Hofmann H, Pyrc K, van der Hoek L, Geier M, Berkhout B, Pöhlmann S. Human coronavirus NL63 employs the severe acute respiratory syndrome coronavirus receptor for cellular entry. Proceedings of the National Academy of Sciences. 2005;102(22):7988.

60. Yeager CL, Ashmun RA, Williams RK, Cardellichio CB, Shapiro LH, Look AT, et al. Human aminopeptidase $\mathrm{N}$ is a receptor for human coronavirus 229E. Nature. 1992 Jun 4;357(6377):420-2.

61. Liu C, Tang J, Ma Y, Liang X, Yang Y, Peng G, et al. Receptor usage and cell entry of porcine epidemic diarrhea coronavirus. Journal of Virology. 2015;89(11):6121.

62. Shirato K, Maejima M, Islam MT, Miyazaki A, Kawase M, Matsuyama S, et al. Porcine aminopeptidase $\mathrm{N}$ is not a cellular receptor of porcine epidemic diarrhea virus, but promotes its infectivity via aminopeptidase activity. 2016;97(10):2528-39. 
63. Szczepanski A, Owczarek K, Bzowska M, Gula K, Drebot I, Ochman M, et al. Canine respiratory coronavirus, bovine coronavirus, and human coronavirus OC43: receptors and attachment factors. Viruses. 2019;11(4):328.

64. Collins AR. HLA Class I antigen serves as a receptor for human coronavirus OC43. Immunological Investigations. 1993;22(2):95-103.

65. Chan CM, Lau SKP, Woo PCY, Tse H, Zheng B-J, Chen L, et al. Identification of Major Histocompatibility Complex Class I C molecule as an attachment factor that facilitates coronavirus HKU1 spike-mediated infection. Journal of Virology. 2009;83(2):1026.

66. Meyerholz DK, Lambertz AM, McCray PB, Jr. Dipeptidyl peptidase 4 distribution in the human respiratory tract: implications for the Middle East respiratory syndrome. The American Journal of Pathology. 2016 Jan;186(1):78-86.

67. Li W, Moore MJ, Vasilieva N, Sui J, Wong SK, Berne MA, et al. Angiotensin-converting enzyme 2 is a functional receptor for the SARS coronavirus. Nature. 2003 Nov 27;426(6965):450-4.

68. Zhang H, Penninger JM, Li Y, Zhong N, Slutsky AS. Angiotensin-converting enzyme 2 (ACE2) as a SARS-CoV-2 receptor: molecular mechanisms and potential therapeutic target. Intensive Care Medicine. 2020;46(4):586-90.

69. Binn LN, Lazar EC, Keenan KP, Huxsoll DL, Marchwicki RH, Strano AJ. Recovery and characterization of a coronavirus from military dogs with diarrhea. Proceedings Annual Meeting of the United States Animal Health Association. 1974(78):359-66.

70. Wang Y, Ma G, Lu C, Wen H. Detection of canine coronaviruses genotype I and II in raised Canidae animals in China. Berliner und Munchener tierarztliche Wochenschrift. 2006 Jan-Feb;119(12):35-9.

71. Rosa GM, Santos N, Grøndahl-Rosado R, Fonseca FP, Tavares L, Neto I, et al. Unveiling patterns of viral pathogen infection in free-ranging carnivores of northern Portugal using a complementary methodological approach. Comparative Immunology, Microbiology and Infectious Diseases. 2020;69:101432.

72. Zarnke RL, Evermann J, Hoef JMV, McNay ME, Boertje RD, Gardner CL, et al. Serologic survey for canine coronavirus in wolves from Alaska. Journal of Wildlife Diseases. 2001;37(4):740-5.

73. McArdle F, Bennett M, Gaskell RM, Tennant B, Kelly DF, Gaskell CJ. Induction and enhancement of feline infectious peritonitis by canine coronavirus. American Journal of Veterinary Research. 1992;53(9):1500-6.

74. Poland AM, Vennema H, Foley JE, Pedersen NC. Two related strains of feline infectious peritonitis virus isolated from immunocompromised cats infected with a feline enteric coronavirus. Journal of Clinical Microbiology. 1996;34(12):3180-4.

75. Vennema H, Poland A, Foley J, Pedersen NC. Feline infectious peritonitis viruses arise by mutation from endemic feline enteric coronaviruses. Virology. 1998 1998/03/30/;243(1):150-7.

76. Evermann JF, Heeney JL, Roelke ME, McKeirnan AJ, O'Brien SJ. Biological and pathological consequences of feline infectious peritonitis virus infection in the cheetah. Archives of virology. 1988;102(3-4):155-71.

77. Mwase M, Shimada K, Mumba C, Yabe J, Squarre D, Madarame H. Positive immunolabelling for feline infectious peritonitis in an African lion (Panthera leo) with bilateral panuveitis. Journal of comparative pathology. 2015 Feb-Apr;152(2-3):265-8.

78. Foley JE, Swift P, Fleer KA, Torres S, Girard YA, Johnson CK. Risk factors for exposure to feline pathogens in California mountain lions (Puma concolor). Journal of Wildlife Diseases. 2013 Apr;49(2):279-93.

79. Horzinek MC, Osterhaus AD, Wirahadiredja RM, de Kreek P. Feline infectious peritonitis (FIP) virus. III. Studies on the multiplication of FIP virus in the suckling mouse. Zentralblatt fur Veterinarmedizin Reihe B. 1978 Dec;25(10):806-15.

80. Woo PCY, Wang M, Lau SKP, Xu H, Poon RWS, Guo R, et al. Comparative analysis of twelve genomes of three novel group $2 \mathrm{c}$ and group $2 \mathrm{~d}$ coronaviruses reveals unique group and subgroup features. Journal of Virology. 2007;81(4):1574. 
233 81. Lau SKP, Li KSM, Tsang AKL, Shek C-T, Wang M, Choi GKY, et al. Recent transmission of a 234 novel alphacoronavirus, bat coronavirus HKU10, from Leschenault's rousettes to Pomona leaf-nosed 235 bats: first evidence of interspecies transmission of coronavirus between bats of different suborders. 236 Journal of Virology. 2012;86(21):11906.

237 82. Wise AG, Kiupel M, Maes RK. Molecular characterization of a novel coronavirus associated with epizootic catarrhal enteritis (ECE) in ferrets. Virology. 2006;349(1):164-74.

83. Garner MM, Ramsell K, Morera N, Juan-Sallés C, Jiménez J, Ardiaca M, et al. Clinicopathologic features of a systemic coronavirus-associated disease resembling feline infectious peritonitis in the domestic ferret (Mustela putorius). Veterinary Pathology. 2008;45(2):236-46.

84. $\mathrm{Xu} \mathrm{Y}$. Genetic diversity and potential recombination between ferret coronaviruses from European and American lineages. Journal of Infection. 2020;80(3):350-71.

85. Tao Y, Shi M, Chommanard C, Queen K, Zhang J, Markotter W, et al. Surveillance of bat coronaviruses in Kenya identifies relatives of human coronaviruses NL63 and 229E and their recombination history. Journal of Virology. 2017;91(5):e01953-16.

86. Fouchier RAM, Hartwig NG, Bestebroer TM, Niemeyer B, de Jong JC, Simon JH, et al. A previously undescribed coronavirus associated with respiratory disease in humans. Proceedings of the National Academy of Sciences of the United States of America. 2004;101(16):6212-6.

87. El-Duah P, Meyer B, Sylverken A, Owusu M, Gottula LT, Yeboah R, et al. Development of a whole-virus ELISA for serological evaluation of domestic livestock as possible hosts of human coronavirus NL63. Viruses. 2019;11(1):43.

88. Pfefferle S, Oppong S, Drexler JF, Gloza-Rausch F, Ipsen A, Seebens A, et al. Distant relatives of severe acute respiratory syndrome coronavirus and close relatives of human coronavirus 229E in bats, Ghana. Emerg Infect Dis. 2009;15(9):1377-84.

89. Corman VM, Baldwin HJ, Tateno AF, Zerbinati RM, Annan A, Owusu M, et al. Evidence for an ancestral association of Human Coronavirus 229E with bats. Journal of Virology. 2015;89(23):11858-70.

90. Crossley BM, Mock RE, Callison SA, Hietala SK. Identification and characterization of a novel alpaca respiratory coronavirus most closely related to the human coronavirus 229E. Viruses. 2012;4(12):3689-700.

91. Barlough JE, Johnson-Lussenburg CM, Stoddart CA, Jacobson RH, Scott FW. Experimental inoculation of cats with human coronavirus 229E and subsequent challenge with feline infectious peritonitis virus. Canadian Journal of Comparative Medicine. 1985;49(3):303-7.

92. Tang XC, Zhang JX, Zhang SY, Wang P, Fan XH, Li LF, et al. Prevalence and genetic diversity of coronaviruses in bats from China. Journal of Virology. 2006;80(15):7481-90.

93. Banerjee A, Kulcsar K, Misra V, Frieman M, Mossman K. Bats and coronaviruses. Viruses. 2019;11(1):41.

94. Yang Y-L, Qin P, Wang B, Liu Y, Xu G-H, Peng L, et al. Broad cross-species infection of cultured cells by bat HKU2-related swine acute diarrhea syndrome coronavirus and identification of its replication in murine dendritic cells in vivo highlight its potential for diverse interspecies transmission. Journal of Virology. 2019;93(24):e01448-19.

95. Lau SKP, Woo PCY, Li KSM, Tsang AKL, Fan RYY, Luk HKH, et al. Discovery of a novel coronavirus, China rattus coronavirus HKU24, from Norway rats supports the murine origin of Betacoronavirus 1 and has implications for the ancestor of Betacoronavirus lineage A. Journal of Virology. 2015;89(6):3076-92.

96. Storz J, Stine L, Liem A, Anderson GA. Coronavirus isolation from nasal swab samples in cattle with signs of respiratory tract disease after shipping. Journal of the American Veterinary Medical Association. 1996 May 1;208(9):1452-5.

97. Ismail MM, Cho KO, Ward LA, Saif LJ, Saif YM. Experimental bovine coronavirus in turkey poults and young chickens. Avian diseases. 2001 Jan-Mar;45(1):157-63.

98. Patrono LV, Samuni L, Corman VM, Nourifar L, Röthemeier C, Wittig RM, et al. Human coronavirus OC43 outbreak in wild chimpanzees, Côte d'Ivoire, 2016. Emerging Microbes and Infections. 2018;7(1):118-. 
$28599 . \quad$ Jacomy H, Talbot PJ. Vacuolating encephalitis in mice infected by human coronavirus OC43. 286 Virology. 2003;315(1):20-33.

287 100. Wang W, Lin X-D, Guo W-P, Zhou R-H, Wang M-R, Wang C-Q, et al. Discovery, diversity and evolution of novel coronaviruses sampled from rodents in China. Virology. 2015;474:19-27. 101. Anthony SJ, Gilardi K, Menachery VD, Goldstein T, Ssebide B, Mbabazi R, et al. Further evidence for bats as the evolutionary source of Middle East respiratory syndrome coronavirus. mBio. 2017;8(2):e00373-17.

102. Reusken CBEM, Raj VS, Koopmans MP, Haagmans BL. Cross host transmission in the emergence of MERS coronavirus. Current Opinion in Virology. 2016;16:55-62.

103. Adney DR, Bielefeldt-Ohmann H, Hartwig AE, Bowen RA. Infection, replication, and transmission of Middle East respiratory syndrome coronavirus in alpacas. Emerg Infect Dis. 2016;22(6):1031-7.

104. Raj VS, Smits SL, Provacia LB, van den Brand JMA, Wiersma L, Ouwendijk WJD, et al. Adenosine deaminase acts as a natural antagonist for dipeptidyl peptidase 4-mediated entry of the Middle East respiratory syndrome coronavirus. Journal of Virology. 2014;88(3):1834.

105. Falzarano D, de Wit E, Feldmann F, Rasmussen AL, Okumura A, Peng X, et al. Infection with MERS-CoV causes lethal pneumonia in the common marmoset. PLoS Pathog. 2014;10(8):e1004250.

106. Johnson RF, Via LE, Kumar MR, Cornish JP, Yellayi S, Huzella L, et al. Intratracheal exposure of common marmosets to MERS-CoV Jordan-n3/2012 or MERS-CoV EMC/2012 isolates does not result in lethal disease. Virology. 2015 2015/11/01/;485:422-30.

107. de Wit E, Prescott J, Baseler L, Bushmaker T, Thomas T, Lackemeyer MG, et al. The Middle East respiratory syndrome coronavirus (MERS-CoV) does not replicate in Syrian hamsters. PLoS One. 2013;8(7):e69127-e.

108. Vergara-Alert J, van den Brand JMA, Widagdo W, Muñoz Mt, Raj S, Schipper D, et al. Livestock susceptibility to infection with Middle East respiratory syndrome coronavirus. Emerg Infect Dis. 2017;23(2):232-40.

109. Cockrell AS, Peck KM, Yount BL, Agnihothram SS, Scobey T, Curnes NR, et al. Mouse dipeptidyl peptidase 4 is not a functional receptor for Middle East respiratory syndrome coronavirus infection. Journal of Virology. 2014;88(9):5195.

110. de Wit E, Rasmussen AL, Falzarano D, Bushmaker T, Feldmann F, Brining DL, et al. Middle East respiratory syndrome coronavirus (MERS-CoV) causes transient lower respiratory tract infection in rhesus macaques. Proceedings of the National Academy of Sciences of the United States of America. 2013;110(41):16598-603.

111. Yao Y, Bao L, Deng W, Xu L, Li F, Lv Q, et al. An animal model of MERS produced by infection of rhesus macaques with MERS coronavirus. The Journal of Infectious Diseases. 2013;209(2):236-42.

112. Haagmans BL, van den Brand JMA, Provacia LB, Raj VS, Stittelaar KJ, Getu S, et al. Asymptomatic Middle East respiratory syndrome coronavirus infection in rabbits. Journal of Virology. 2015;89(11):6131-5.

113. Li W, Shi Z, Yu M, Ren W, Smith C, Epstein JH, et al. Bats are natural reservoirs of SARS-like coronaviruses. Science. 2005;310(5748):676-9.

114. Wang L-F, Shi Z, Zhang S, Field H, Daszak P, Eaton BT. Review of bats and SARS. Emerg Infect Dis. 2006;12(12):1834-40.

115. Hu B, Ge X, Wang L-F, Shi Z. Bat origin of human coronaviruses. Virology Journal. 2015 2015/12/22;12(1):221.

116. Ming W, Meiying Y, Huifang X, Weili L, Biao K, Bojian Z, et al. SARS-CoV infection in a restaurant from palm civet. Emerg Infect Dis. 2005;11(12):1860.

117. Weingartl HM, Copps J, Drebot MA, Marszal P, Smith G, Gren J, et al. Susceptibility of pigs and chickens to SARS coronavirus. Emerg Infect Dis. 2004;10(2):179-84.

118. WHO. Consensus document on the epidemiology of severe acute respiratory syndrome (SARS): World Health Organization; 2003. 
336 119. Fouchier RAM, Kuiken T, Schutten M, van Amerongen G, van Doornum GJJ, van den 337 Hoogen BG, et al. Koch's postulates fulfilled for SARS virus. Nature. 2003 2003/05/01;423(6937):240-. 338 120. Xu R-H, He J-F, Evans MR, Peng G-W, Field HE, Yu D-W, et al. Epidemiologic clues to SARS 339 origin in China. Emerg Infect Dis. 2004;10(6):1030-7.

340 121. Lee SH. The SARS epidemic in Hong Kong. Journal of epidemiology and community health. 3412003 Sep;57(9):652-4.

342 122. Roberts A, Vogel L, Guarner J, Hayes N, Murphy B, Zaki S, et al. Severe acute respiratory 343 syndrome coronavirus infection of golden syrian hamsters. Journal of Virology. 2005;79(1):503.

123. Wu D, Tu C, Xin C, Xuan H, Meng Q, Liu Y, et al. Civets are equally susceptible to experimental infection by two different severe acute respiratory syndrome coronavirus isolates. Journal of Virology. 2005;79(4):2620.

124. Roberts A, Paddock C, Vogel L, Butler E, Zaki S, Subbarao K. Aged BALB/c mice as a model for increased severity of severe acute respiratory syndrome in elderly humans. Journal of Virology. 2005;79(9):5833.

125. Qin C, Wang J, Wei Q, She M, Marasco WA, Jiang H, et al. An animal model of SARS produced by infection of Macaca mulatta with SARS coronavirus. The Journal of Pathology. 2005;206(3):251-9.

126. Rockx B, Kuiken T, Herfst S, Bestebroer T, Lamers MM, Oude Munnink BB, et al. Comparative pathogenesis of COVID-19, MERS, and SARS in a nonhuman primate model. Science. 2020:eabb7314.

127. Zhu N, Zhang D, Wang W, Li X, Yang B, Song J, et al. A novel coronavirus from patients with pneumonia in China, 2019. New England Journal of Medicine. 2020;382(8):727-33.

128. Bao L, Deng W, Huang B, Gao H, Liu J, Ren L, et al. The pathogenicity of SARS-CoV-2 in hACE2 transgenic mice. Nature. 2020.

129. Munster VJ, Feldmann F, Williamson BN, van Doremalen N, Pérez-Pérez L, Schulz J, et al. Respiratory disease in rhesus macaques inoculated with SARS-CoV-2. Nature. 2020 2020/05/12.

130. AVMA. Coronaviruses in domestic species. In: AVMA, editor.; 2020.

131. USDA. Veterinary Biological Products. Ames, IA, USA: U.S. Department of Agriculture APHIS VS; 2020.

132. Levy JK. Overview of Feline Infectious Peritonitis. Merck Veterinary Manual: Merck; 2014.

133. Pedersen NC. An update on feline infectious peritonitis: diagnostics and therapeutics. The Veterinary Journal. 2014;201(2):133-41.

134. Morrisey JK. Infectious Diseases of Ferrets. Merck Veterinary Manual; 2013.

135. Abdul-Rasool S, Fielding BC. Understanding human coronavirus HCoV-NL63. Open Virol J. 2010;4:76-84.

136. CDC. Common Human Coronaviruses. Coronavirus 2020 February 13, 2020 [cited July 13, 2020]; Available from: https://www.cdc.gov/coronavirus/general-information.html

137. Crossley BM, Barr BC, Magdesian KG, Ing M, Mora D, Jensen D, et al. Identification of a novel coronavirus possibly associated with acute respiratory syndrome in alpacas (Vicugna Pacos) in California, 2007. Journal of Veterinary Diagnostic Investigation. 2010;22(1):94-7.

138. McIntosh K. Coronaviruses. 2020 February 182020 [cited 2020; Available from: https://www.uptodate.com/contents/coronaviruses

139. Song D, Moon H, Kang B. Porcine epidemic diarrhea: a review of current epidemiology and available vaccines. Clin Exp Vaccine Res. 2015;4(2):166-76.

140. Zhang F, Luo S, Gu J, Li Z, Li K, Yuan W, et al. Prevalence and phylogenetic analysis of porcine diarrhea associated viruses in southern China from 2012 to 2018. BMC Veterinary Research. 2019 2019/12/27;15(1):470.

141. Esper F, Weibel C, Ferguson D, Landry ML, Kahn JS. Coronavirus HKU1 infection in the United States. Emerg Infect Dis. 2006;12(5):775-9.

142. Baseler L, de Wit E, Feldmann H. A comparative review of animal models of Middle East respiratory syndrome coronavirus infection. Veterinary Pathology. 2016 2016/05/01;53(3):521-31. 
143. Precisionvaccinations. Coronavirus Vaccines. 2020 [cited 2020; Available from: https://www.precisionvaccinations.com/vaccines/coronavirus-vaccines

389 144. Molenaar RJ, Vreman S, Hakze-van der Honing RW, Zwart R, de Rond J, Weesendorp E, et 390 al. Clinical and pathological findings in SARS-CoV-2 disease outbreaks in farmed mink. Veterinary 391 Pathology. 2020:300985820943535.

392

(C) 2020 by the authors. Submitted for possible open access publication under the terms and conditions of the Creative Commons Attribution (CC BY) license (http://creativecommons.org/licenses/by/4.0/).

393 